\title{
A Blind Deconvolution Approach to Discontinuity Location and Characterization in Ultrasonic Nondestructive Testing
}

\author{
Giovanni A. Guarneri*, Daniel R. Pipa ${ }^{\dagger}$, Flávio Neves Junior ${ }^{\dagger}$ and Lúcia V. R. de Arruda ${ }^{\dagger}$ \\ ${ }^{*}$ Graduate Program in Electrical Engineering (PPGEE) \\ Federal University of Technology - Paraná (UTFPR), Pato Branco, Paraná, Brazil \\ Email: giovanni@utfpr.edu.br \\ ${ }^{\dagger}$ Graduate Program in Electrical and Computer Engineering (CPGEI) \\ Federal University of Technology - Paraná (UTFPR), Curitiba, Paraná, Brazil \\ Email: see http://www.utfpr.edu.br/curitiba/estrutura-universitaria/diretorias/dirppg/programas/cpgei/professores
}

\begin{abstract}
This paper presents a new algorithm for discontinuity location and characterization using $A$-scan signals from an ultrasonic inspection system. The algorithm is based on solving an inverse problem in which the observation model is different from that traditionally used. In this model, the input vector represents the location of the geometrical center of the discontinuity and the scattering amplitude of the discontinuity is embedded in the impulse response of the ultrasonic inspection system. First, we jointly estimate the locations and the scattering amplitudes of the discontinuities from the acquired signals. Then, the geometrical parameters of the discontinuities are calculated from the estimated scattering amplitude. The method is tested to characterize side-drilled holes using both synthetic and real data. The results demonstrates the effectiveness of the algorithm.
\end{abstract}

\section{INTRODUCTION}

Discontinuity characterization in solid objects by ultrasonic nondestructive evaluation (NDE) comprises finding unknown parameters such as flaw type, size, orientation and location. Such evaluation is of central importance to assure the structural integrity in materials and components of the mechanical structures [1]. The presence of discontinuities in these structures may or may not be acceptable depending on whether they degrade or not the performance or durability of the structure [2].

The discontinuity characterization from A-scan signals collected by an ultrasonic inspection system (UIS) has already developed using several approaches. In general, these approaches use $A$-scan signals collected by UIS with: (I) a monostatic transducer in pulse-echo configuration using Synthetic Aperture Focusing Technique (SAFT) [1], [3]; (II) two transducers using Time of Flight Diffraction (TOFD) technique [4]; (III) an array of transducers using Total Focusing Method (TFM) or Full Matrix Capture (FMC) [5].

In [6], SAFT is used to collect A-scan signals and the size of side-drilled holes (SDH) is determined by evaluating the traveling distance of the reflected signal. In [3], a comparative study of the SAFT and TOFD techniques is presented to sizing several refletors. The use of ultrassonic arrays to characterize

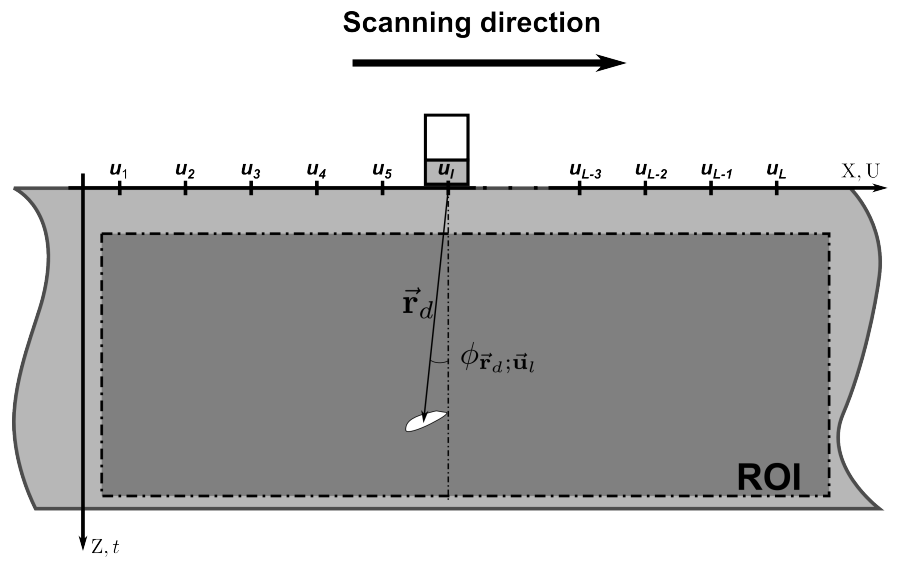

Figure 1. A linear scan to carry out an ultrasonic NDE on an object with a discontinuity. The transducer is placed through a set of $L$ different positions along a direction from $u_{1}$ to $u_{L}$. The spatial sampling period must be constant. An A-scan signal is acquired at each position.

flaws is presented in several papers. In [7], the measurement of the scattering coefficient matrix is used to obtain the size, shape and orientation of a crack-like defect. In [8], the correlation coefficient and the structural similarity of scattering matrices are used for defect characterization.

In this paper, we present a novel algorithm to estimate both the location and the parameters of the discontinuities in solid objects based on solving an inverse problem. Differently from traditional approaches where the input of the model represents the internal acoustic reflexivity [9]-[11], our observation model uses the location of the geometrical center of the discontinuity as input. In addition, the scattering amplitude of the discontinuity is embedded in the impulse response of the system. Thus, the proposed algorithm is able to estimate from the measured $A$-scan signals the location of the geometrical center of the discontinuity and also its parameters simultaneously. 
Table I

SCATTERING AMPLITUDE EXPRESSIONS IN KIRCHHOFF APPROXIMATION FOR SOME DISCONTINUITIES

\begin{tabular}{|c|c|}
\hline Discontinuity & Scattering amplitude $S_{A}(\omega, \Theta)$ \\
\hline $\begin{array}{c}\text { Spherical } \\
\text { void }\end{array}$ & $\begin{array}{c}-\frac{b}{2} \exp (-\mathrm{i} k b)\left[\exp (-\mathrm{i} k b)-\frac{\sin (k b)}{k b}\right] \\
\Theta=(b), \\
\text { where } b \text { is the radius of sphere }\end{array}$ \\
\hline $\begin{array}{l}\text { Side-drilled } \\
\text { hole (SDH) }\end{array}$ & $\begin{array}{c}\frac{k b D}{2}\left[\mathrm{~J}_{1}(2 k b)-\mathrm{iS}_{1}(2 k b)\right]+\frac{\mathrm{i} k b D}{\pi} \\
\Theta=(b, D), \\
\text { where } b \text { is the radius of SDH } \\
\text { and } D \text { is its length }\end{array}$ \\
\hline $\begin{array}{l}\text { Circular flat } \\
\text { crack }\end{array}$ & $\begin{array}{l}\mathrm{i} k b^{2} \cos \alpha \operatorname{jinc}(2 k b \sin \alpha) \\
\boldsymbol{\Theta}=(b, \alpha), \\
\text { where } b \text { is the radius of crack } \\
\text { and } \alpha \text { is the angle between incident wave } \\
\text { and the unit normal to the crack }\end{array}$ \\
\hline $\begin{array}{l}\text { Infinitesimal } \\
\text { point }\end{array}$ & 1 \\
\hline
\end{tabular}

\section{OBSERVATION MODEL}

An UIS can be modeled as a linear time invariant (LTI) system [12]. This system has a frequency response composed by a concatenation of several LTI systems, each one representing a part of the UIS. Conveniently, only the scattering amplitude depends directly on the physical and geometrical properties of a discontinuity [12]. The scattering amplitude is referred to as $S_{A}(\omega, \boldsymbol{\Theta})$ in this paper, where $\omega$ is the angular frequency and $\Theta$ is a vector containing the discontinuities parameters. The expressions for $S_{A}(\omega, \Theta)$ depend on the type of discontinuity. They can be defined as parametric expressions in which the parameters are related to the dimensions and orientation of the discontinuity [12]. Table I shows the scattering amplitude expressions in the Kirchhoff approximation for some discontinuities [13].

Suppose we have a metallic object with only one discontinuity. This condition is easily obtained in practical situations. The inspector can define a Region of Interest (ROI) in the object and select a sampling window to characterize only one discontinuity. An UIS with a circular piston transducer of radius $a$ carries out a linear scan to collect $L A$-scan signals with $N$ samples each (Fig. 1). This A-scan signals can be modeled in the frequency domain by Eq. (1) [11]:

$$
\begin{aligned}
V_{o}\left(\overrightarrow{\mathbf{u}}_{l}, \omega\right) & =\left(-2 a^{2} \mathrm{i} k\right) H_{e}^{2}(\omega) S_{A}(\omega, \boldsymbol{\Theta}) \\
& \times \int_{\overrightarrow{\mathbf{r}} \in \text { ROI }} \operatorname{jinc}^{2}\left[k a \sin \phi_{\left.\overrightarrow{\mathbf{r}} ; \overrightarrow{\mathbf{u}}_{l}\right]}\right] \exp \left(\mathrm{i} 2 k\left|\overrightarrow{\mathbf{r}}-\overrightarrow{\mathbf{u}}_{l}\right|\right) f(\overrightarrow{\mathbf{r}}) d \overrightarrow{\mathbf{r}},
\end{aligned}
$$

where $\overrightarrow{\mathbf{u}}_{l}$ is the central point of the active surface of transducer during linear scanning; $k=\omega / c$ is the spatial frequency or wavenumber ( $c$ is the sound speed of longitudinal waves into propagation medium); $H_{e}(\omega)$ is the combined frequency response of all electrical circuits, plus electrical-mechanical conversion in transducer; $\overrightarrow{\mathbf{r}}_{d}$ is the position of the geometrical center of the discontinuity; $\phi_{\overrightarrow{\mathbf{r}}_{d} ; \overrightarrow{\mathbf{u}}_{l}}$ is the angle between normal axis of transducer surface and the vector $\overrightarrow{\mathbf{r}}-\overrightarrow{\mathbf{u}}_{l}$; $\operatorname{jinc}(x)=$ $\mathrm{J}_{1}(x) / x, \mathrm{~J}_{1}(\cdot)$ is a first order Bessel function [12].

The function $f(\overrightarrow{\mathbf{r}})$ is defined by:

$$
f(\overrightarrow{\mathbf{r}})=\left\{\begin{array}{ll}
1, & \text { for } \overrightarrow{\mathbf{r}}=\overrightarrow{\mathbf{r}}_{d} \\
0, & \text { for all other points in the ROI }
\end{array},\right.
$$

which represents the position of geometrical center of the discontinuity modeled by $S_{A}(\omega, \boldsymbol{\Theta})$. The domain of $f(\overrightarrow{\mathbf{r}})$ are all points in ROI. This is a novel approach to flaw characterization and location.

Eq. (2) defines $f(\overrightarrow{\mathbf{r}})$ as a sparse function. Therefore, the model represented by Eq. (1) is similar to the model present in [11, Eq. 7]. Thus, following the same procedures of [11], it is possible to represent Eq. (1) (in the time and the space domains and assuming a 2D ROI) in matrix format:

$$
\mathbf{v}=\underbrace{\mathbf{F}^{\dagger} \mathbf{B}_{A}(\boldsymbol{\Theta}) \mathbf{S}^{\dagger} \mathbf{F}}_{\mathbf{H}(\boldsymbol{\Theta})} \mathbf{f}+\boldsymbol{\eta},
$$

where $\mathbf{v}$ e $\mathbf{f}$ are, respectively, the samples of $A$-scan signals and the discrete representation of $f(\overrightarrow{\mathbf{r}})$, both stacked as column vectors; $\mathbf{F}$ is the 2D Discrete Fourier Transform (DFT) matrix; $\mathbf{S}^{\dagger}$ is the Stolt modeling matrix [11]; $\mathbf{B}_{A}(\boldsymbol{\Theta})=\operatorname{diag}\left[\left(-2 a^{2} \mathrm{i} k\right) H_{e}^{2}(\omega) \operatorname{jinc}^{2}\left(k_{u} a / 2\right) S_{A}(\omega, \boldsymbol{\Theta})\right]$ includes the combined frequency response of all electrical circuits, the diffraction effect of circular transducer and the scattering amplitude expression of the discontinuity; and $\boldsymbol{\eta}$ is the vector representing the uncertainties in the measurements during data acquisition process by UIS.

\section{DISCONTINUITY CHARACTERIZATION PROBLEM}

The recast of Eq. (1) into Eq. (3) indicates that the discontinuity characterization problem can be treated as a blind deconvolution problem [14]. The discontinuity location (vector f) and its parameters (embedded in $\mathbf{H}(\boldsymbol{\Theta})$ model) should be estimated from A-scan signals measured by UIS. In a blind deconvolution problem, both the input and the model may be partially or totally unknown. Therefore, some kind of prior information about the input and/or the model, such as physical 
characteristics or statistical information, is necessary to solve the problem [14].

Although the vector $\mathbf{f}$ is generally unknown in a practical discontinuity characterization problem, it can be safely modeled as a sparse vector as one expects to find few discontinuities in a specimen.

\section{A. Algorithm description}

As already state, an algorithm to solve this problem should be able to find both the discontinuity location in ROI and the parameters $\Theta$ of scattering amplitude expression embedded in model $\mathbf{H}(\Theta)$. Using Eq. (3) and a penalized optimization approach [15], the proposed algorithm is defined as:

$$
\begin{aligned}
\{\hat{\mathbf{f}}, \hat{\boldsymbol{\Theta}}\} & =\arg \min _{\mathbf{f}, \boldsymbol{\Theta}} Q(\mathbf{f} ; \boldsymbol{\Theta}) \text { where } \\
Q(\mathbf{f} ; \boldsymbol{\Theta}) & =\|\mathbf{v}-\mathbf{H}(\boldsymbol{\Theta}) \mathbf{f}\|_{2}^{2}+\lambda\|\mathbf{f}\|_{1} .
\end{aligned}
$$

The cost function (4) has two terms. The first ensures fidelity of the solution with measured data. It corresponds to the Nonlinear Least Squares method [16] because the expressions of the scattering amplitudes for different types of discontinuities are nonlinear with respect to $\Theta$ [12]. The second is a regularization term that promotes the sparsity of $\hat{\mathbf{f}}$ [15], [17]. The regularization parameter $\lambda$ controls the tradeoff between the two terms [18].

There is a difficulty to solve Eq. (4a) because we need to estimate two distinct variables, $\mathbf{f}$ and $\Theta$. A widely used approach to solve this kind of problem is the Alternating Minimization (AM) [17]. In this method, we solve the problem with respect to one unknown while holding the other unknown constant. There are some well-known shortcomings applying $\mathrm{AM}$ in the discontinuity characterization problem: (I) we need a strategy to choose initial values of $\Theta$; (II) the algorithm must reconstruct $\mathbf{f}$ at each iteration and this can be computationally expensive; (III) we need define a stopping criteria which terminates the algorithm when $\hat{\mathbf{f}}$ and $\hat{\boldsymbol{\Theta}}$ approximations are sufficiently high quality [19]. The proposed algorithm, referred to as Ultrasonic Discontinuity Characterization (UTDisC), is based on the AM method. The pseudocode of UTDisC is shown in Fig. 2.

Let $\mathcal{T}$ be the domain of $\Theta$, including all possible values within acceptable limits. We select a finite subset $\left\{\boldsymbol{\Theta}_{1}, \boldsymbol{\Theta}_{2}, \cdots, \boldsymbol{\Theta}_{K}\right\}$. For each $\boldsymbol{\Theta}_{i}$ in this subset, we evaluate $\mathbf{H}\left(\boldsymbol{\Theta}_{i}\right)$. Given $\mathbf{H}=\mathbf{H}\left(\boldsymbol{\Theta}_{i}\right)$, we can find $\hat{\mathbf{f}}$ using any algorithm for sparse image reconstruction such as UTSR [11]. However, we chose the backprojection algorithm, defined in Eq. (5), to decrease computational cost.

$$
\hat{\mathbf{f}}_{B P}=\mathbf{H}^{\dagger} \mathbf{v}
$$

Although $\hat{\mathbf{f}}_{B P}$ is blurrier than $\hat{\mathbf{f}}$ calculated by UTSR algorithm, it can be used to estimate $\hat{\boldsymbol{\Theta}}$. The rationale is that the higher amplitude point in $\hat{\mathbf{f}}_{B P}$ indicates the discontinuity central location in ROI with a sufficient precision. We do not need define a stopping criteria because there are not iterations. Finally, we estimate a better approximation of $\mathbf{f}$ using the UTSR algorithm and $\mathbf{H}(\hat{\boldsymbol{\Theta}})$.

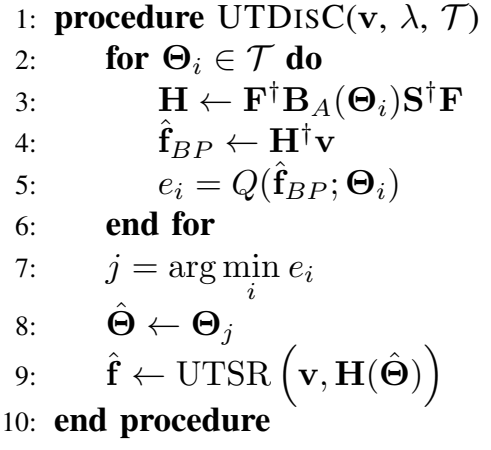

Figure 2. Pseudocode of the proposed algorithm to solve the discontinuity characterization problem based on Nonlinear Least Squares with sparse restriction.

\section{RESULTS}

\section{A. Simulations}

Initially, we test the UTDisC algorithm using synthetic data generated by Eq. (1). We add white Gaussian noise with SNR of $20 \mathrm{~dB}$ to the synthetic A-scan signals. We consider only SDH discontinuities. The expression for scattering amplitude of the side-drilled hole in the Kirchhoff approximation is [13, Eq. 10.53]

$$
S_{A}(\omega, b, D)=\frac{k b D}{2}\left[\mathrm{~J}_{1}(2 k b)-\mathrm{iS}_{1}(2 k b)\right]+\frac{\mathrm{i} k b D}{\pi}
$$

where $D$ is the length of $\mathrm{SDH} ; b$ is the radius of $\mathrm{SDH} ; k$ is the wavenumber; $\mathrm{J}_{1}(\cdot)$ is a Bessel function of order one and $\mathrm{S}_{1}(\cdot)$ is a Struve function of order one. As $S_{A}(\omega, b, D)$ is directly proportional to $D$, it is possible to treat $D$ as an attenuating term that is offset by the UIS. Therefore, we consider the $S_{A}(\omega, b, D)$ expression normalized by $D$ and $b$ is the only parameter in the vector $\Theta$. We generate synthetic data for SDHs of diameters $1 \mathrm{~mm}, 5 \mathrm{~mm}$ and $10 \mathrm{~mm}$ in steel blocks with $c=5860 \mathrm{~m} / \mathrm{s}$. The searching grid for diameter of the SDHs is $0.1 \mathrm{~mm}$ to $15 \mathrm{~mm}$ with steps of $0.1 \mathrm{~mm}$. The value of $\lambda$ is set to $1 \times 10^{-4}$.

The results of applying the UTDisC algorithm to synthetic data are shown in Fig. 3. In Fig. 3a, the ordinate represents the numerical value of Eq. (4b) and the abscissa is the diameter of discontinuities (parameter $\boldsymbol{\Theta}$ ). Highlights indicate the minimum of Eq. (4b) for each SDH. These points correspond to the estimated diameter of the SDHs. The algorithm found the correct values of the diameters for all tested SDHs from synthetic data. The Fig. $3 \mathrm{~b}$ shows the reconstructed image by UTSR algorithm for the SDH of diameter $1 \mathrm{~mm}$. The UTDisC algorithm has located the geometrical center of the SDH at $x=15 \mathrm{~mm}$ and $z=40.02 \mathrm{~mm}$.

\section{B. Experimental results}

The experimental validation of the UTDisC algorithm was carried out with A-scan signals collected by an UIS inspecting three different specimens. Each specimen is a steel block containing a SDH of diameter $1 \mathrm{~mm}, 5 \mathrm{~mm}$ and $10 \mathrm{~mm}$. All SDHs are located at $x=15 \mathrm{~mm}$ and $z=40 \mathrm{~mm}$. The grid 


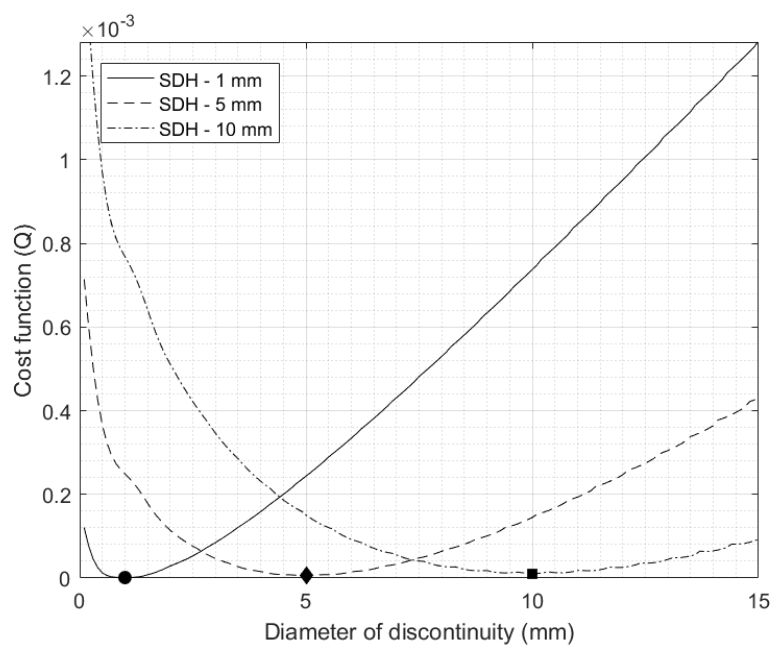

(a)

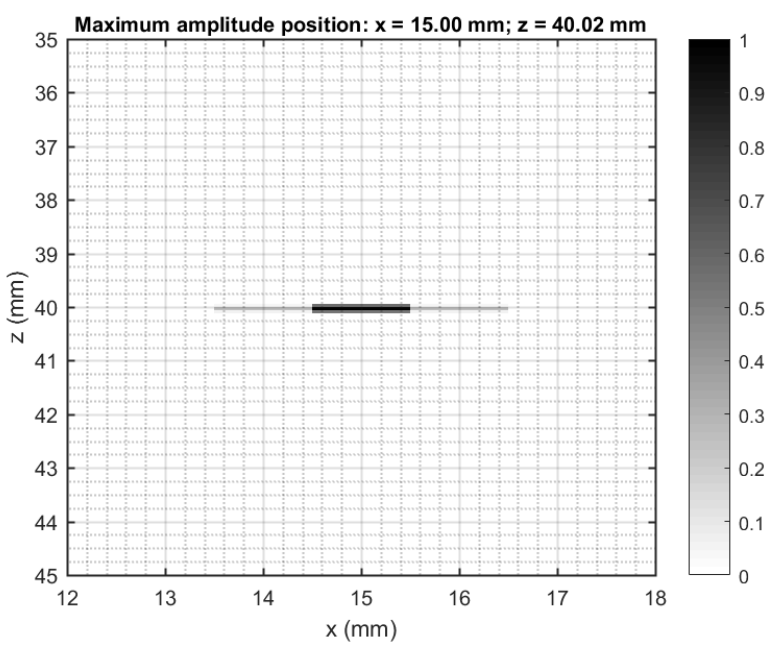

(b)

Figure 3. Simulation results for the UTDisC algorithm. (a) Graphs showing the results of applying the UTDisC algorithm to synthetic data for SDHs of diameter $1 \mathrm{~mm}, 5 \mathrm{~mm}$ and $10 \mathrm{~mm}$. Highlights indicate the minimum of Eq. (4b) for each SDH. (b) The reconstructed image by UTSR algorithm for a simulated SDH of diameter $1 \mathrm{~mm}$ with geometrical center at $x=15 \mathrm{~mm}$ and $z=40 \mathrm{~mm}$. The geometrical center of the SDH was located exactly.

Table II

RESULTS GIVEN BY APPLYING THE UTDISC ALGORITHM TO THE EXPERIMENTAL DATA COLLECTED FROM THREE DIFFERENT SPECIMENS AND VARYING THE REGULARIZATION PARAMETER $\lambda$. EACH CELL PRESENTS THE ESTIMATED DIAMETER OF THE SDHS

\begin{tabular}{|c|c|c|c|}
\hline $\begin{array}{c}\lambda \\
\left(\times 10^{-3}\right)\end{array}$ & \multicolumn{3}{|c|}{ Specimen } \\
\cline { 2 - 4 } & SDH 1 mm & SDH 5 mm & SDH 10 mm \\
\hline 0 & $1.1 \mathrm{~mm}$ & $5.5 \mathrm{~mm}$ & $11.3 \mathrm{~mm}$ \\
\hline 0.1 & $1.1 \mathrm{~mm}$ & $5.5 \mathrm{~mm}$ & $11.3 \mathrm{~mm}$ \\
\hline 0.2 & $1.1 \mathrm{~mm}$ & $5.5 \mathrm{~mm}$ & $11.3 \mathrm{~mm}$ \\
\hline 0.5 & $1.1 \mathrm{~mm}$ & $5.5 \mathrm{~mm}$ & $10.7 \mathrm{~mm}$ \\
\hline 1 & $1.0 \mathrm{~mm}$ & $5.5 \mathrm{~mm}$ & $10.7 \mathrm{~mm}$ \\
\hline 2 & $1.0 \mathrm{~mm}$ & $4.8 \mathrm{~mm}$ & $10.7 \mathrm{~mm}$ \\
\hline 5 & $0.6 \mathrm{~mm}$ & $4.1 \mathrm{~mm}$ & $9.3 \mathrm{~mm}$ \\
\hline 10 & $0.5 \mathrm{~mm}$ & $2.8 \mathrm{~mm}$ & $7.3 \mathrm{~mm}$ \\
\hline 20 & $0.2 \mathrm{~mm}$ & $1.5 \mathrm{~mm}$ & $3.2 \mathrm{~mm}$ \\
\hline 50 & $0.1 \mathrm{~mm}$ & $0.1 \mathrm{~mm}$ & $0.1 \mathrm{~mm}$ \\
\hline
\end{tabular}

search was the same for the test with synthetic data. As $\lambda$ is related to the SNR of the $A$-scan signals acquired by UIS and we do not know it a priori, we repeat the test using different values for $\lambda$. The results are shown in Table II. Each cell presents the estimated diameter of the SDHs.

The UTDisC algorithm presented better results using $\lambda=2 \times 10^{-3}$ as shown in highlighted row in Table II. Fig. 4 presents the results of applying the UTDisC algorithm to experimental data and using $\lambda=2 \times 10^{-3}$. These are presented in the same way of Fig. 3. Comparing Fig. 3a and 4a, we note the similarity in the graphs indicating the validity of the proposed algorithm. We also note that the absolute values of the minimum points of the cost function increase when the diameters of the SDHs are larger. This is caused by the first term of the cost function in Eq. (4) and may be related to two hypotheses: (I) the scattering amplitude expression in Kirchhoff approximation is not proper for SDHs with large diameters; (II) the backprojection algorithm for image reconstruction is not suitable when the diameter of the SDHs increases. We shall investigate these hypotheses in future work. The reconstructed image by UTSR algorithm for a real SDH of diameter $1 \mathrm{~mm}$ is shown in Fig. 4b. The UTDisC algorithm has located the geometrical center of the SDH at $x=15 \mathrm{~mm}$ and $z=40.26 \mathrm{~mm}$. The relative error in SDH depth was below $1 \%$.

\section{CONCLUSion}

In this paper, we propose a new algorithm to estimate the location and the geometrical parameters of discontinuities in solid objects. The proposed algorithm is based on a new $A$ scan observation model, in which the scattering amplitude of the discontinuity is embedded in the impulse response of the UIS. Also, the location of the geometric center of the discontinuity is taken as the stimulus for this system. Thus, the discontinuity characterization problem has two unknown variables: (I) the location of the discontinuity; (II) the discontinuity parameters. We approach it as a blind deconvolution problem.

The proposed algorithm has two steps: (I) identification of the discontinuity parameters in the impulse response of the system; (II) image reconstruction to locate the discontinuity. In the first step, the scatterring amplitude is calculated for each vector of parameters in a grid search. The scattering amplitude is embedded in $\mathbf{H}(\boldsymbol{\Theta})$ and is used to estimate the discontinuity location by the backprojection algorithm in the second step. Finally, we use $\mathbf{H}(\Theta)$ and discontinuity location to evaluate the cost function of an optimization problem. The minimum of this cost function indicates the most suitable solution (parameters and location) to characterize the discontinuity. 


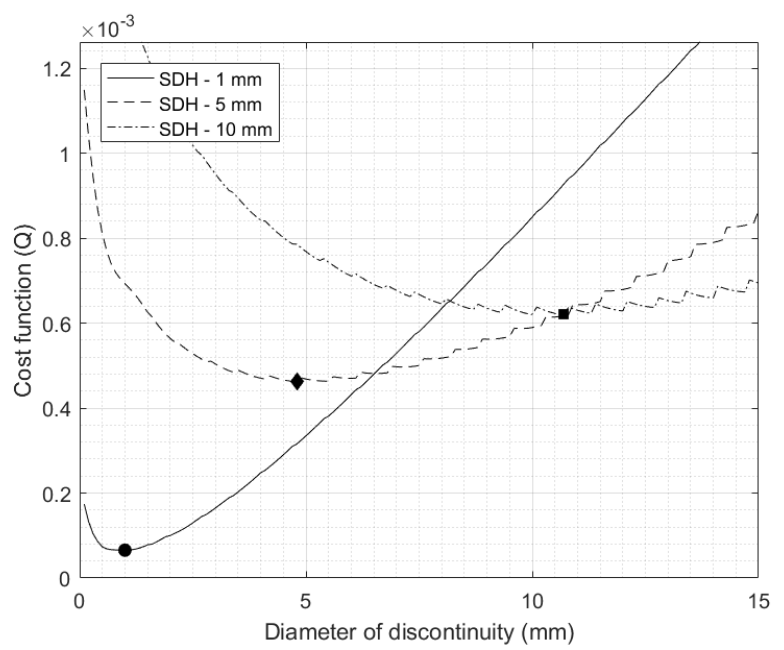

(a)

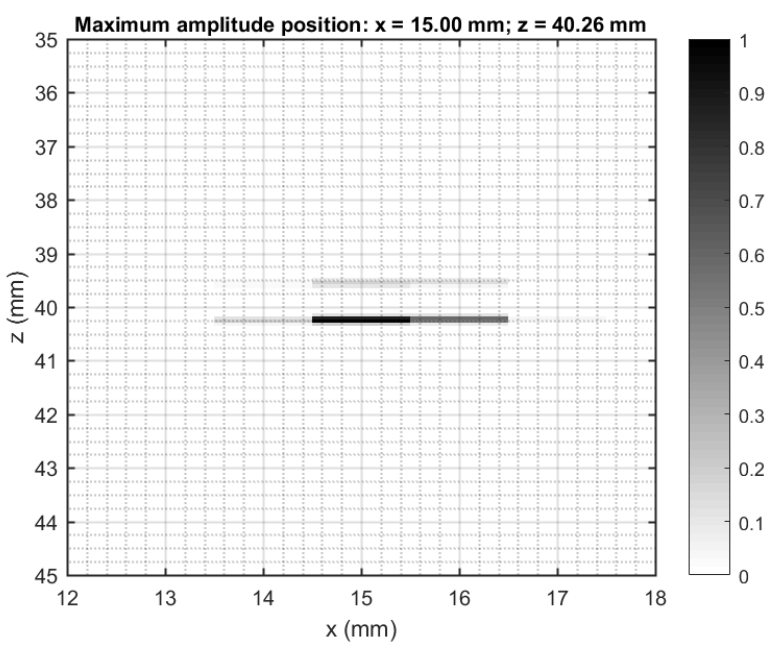

(b)

Figure 4. Experimental results for the UTDisC algorithm. (a) Graphs showing the results of applying the UTDisC algorithm to experimental data for SDHs of diameter $1 \mathrm{~mm}, 5 \mathrm{~mm}$ and $10 \mathrm{~mm}$, with $\lambda=2 \times 10^{-3}$. Highlights indicate the minimum of Eq. (4b) for each SDH. (b) The reconstructed image by UTSR algorithm for a real SDH of diameter $1 \mathrm{~mm}$ located at $x=15 \mathrm{~mm}$ and $z=40 \mathrm{~mm}$. The geometrical center of the SDH was located at $x=15 \mathrm{~mm}$ and $z=40.26 \mathrm{~mm}$. The relative error in SDH depth was below $1 \%$.

The results obtained in the simulations and the experimental validation for characterizing SDH discontinuities demonstrate the effectiveness of the algorithm. Future work includes: (I) application and performance evaluation of the proposed algorithm on specimens containing other types of discontinuities such as cracks and porosities (spherical voids); (II) a performance comparison with other methods for discontinuity characterization.

\section{ACKNOWLEDGMENT}

The authors would like to thank the support of following Brazilian institutions and agencies: UTFPR (campus Pato Branco); ANP, PETROBRAS, FINEP and MCT, through the Human Resources Program of ANP for the oil and gas sector (PRH-ANP/PFRH-PETROBRAS-PRH10/UTFPR); and CNPq (grant 312023/2015-4, 309119/2015-4, 305816/2014-4).

\section{REFERENCES}

[1] R. Thompson and D. Thompson, "Ultrasonics in nondestructive evaluation," Proceedings of the IEEE, vol. 73, no. 12, pp. 1716-1755, Dec 1985.

[2] C. Hellier, Handbook of Nondestructive Evaluation, ser. McGraw-Hill handbooks. New York, NY, USA: Mcgraw-hill, 2003.

[3] J. Prager, J. Kitze, C. Acheroy, D. Brackrock, G. Brekow, and M. Kreutzbruck, "Saft and tofd-a comparative study of two defect sizing techniques on a reactor pressure vessel mock-up," Journal of Nondestructive Evaluation, pp. 1-13, 2012.

[4] S. Mondal and T. Sattar, "An overview tofd method and its mathematical model," NDT.net, vol. 5, no. 4, April 2000. [Online]. Available: http://www.ndt.net/article/v05n04/mondal/mondal.htm

[5] B. W. Drinkwater and P. D. Wilcox, "Ultrasonic arrays for nondestructive evaluation: A review," NDT \& E International, vol. 39, no. 7, pp. $525-541,2006$.

[6] R.-J. Liou, K.-C. Kao, C.-Y. Yeh, and M.-S. Chen, "Flaw detection and sizing of ultrasonic images using wavelet transform and saft," in Intelligent Signal Processing and Communication Systems, 2004. ISPACS 2004. Proceedings of 2004 International Symposium on. IEEE, 2004, pp. 106-110.
[7] D. Zhang, J. Tao, and H. Zheng, "A novel gridding algorithm using nufft with applications to ultrasound diffraction tomography," in Bioinformatics and Biomedical Engineering, 2008. ICBBE 2008. The 2nd International Conference on. IEEE, 2008, pp. 2473-2476.

[8] L. Bai, A. Velichko, and B. W. Drinkwater, "Ultrasonic characterization of crack-like defects using scattering matrix similarity metrics," IEEE transactions on ultrasonics, ferroelectrics, and frequency control, vol. 62 , no. 3 , pp. 545-559, 2015.

[9] F. Lingvall, T. Olofsson, and T. Stepinski, "Synthetic aperture imaging using sources with finite aperture: Deconvolution of the spatial impulse response," The Journal of the Acoustical Society of America, vol. 114, p. 225, 2003.

[10] T. Stepinski, "An implementation of synthetic aperture focusing technique in frequency domain," Ultrasonics, Ferroelectrics and Frequency Control, IEEE Transactions on, vol. 54, no. 7, pp. 1399-1408, 2007.

[11] G. A. Guarneri, D. R. Pipa, F. N. Junior, L. V. R. de Arruda, and M. V. W. Zibetti, "A sparse reconstruction algorithm for ultrasonic images in nondestructive testing," Sensors, vol. 15, no. 4, pp. 9324-9343, 2015. [Online]. Available: http://www.mdpi.com/1424-8220/15/4/9324

[12] L. W. Schmerr, Fundamentals of ultrasonic nondestructive evaluation: a modeling approach. New York, NY, USA: Plenum Press, 1998.

[13] L. W. Schmerr and S.-J. Song, Ultrasonic nondestructive evaluation systems: models and measurements. New York, NY, USA: Springer, 2007.

[14] D. Kundur and D. Hatzinakos, "Blind image deconvolution," Signal Processing Magazine, IEEE, vol. 13, no. 3, pp. 43-64, May 1996.

[15] S. Boyd and L. Vandenberghe, Convex optimization. Cambridge, UK: Cambridge University Press, 2009.

[16] S. M. Kay, Fundamentals of Statistical Signal Processing, Volume I: Estimation Theory. Prentice Hall, 1993.

[17] T. Bishop, S. Babacan, B. Amizic, A. Katsaggelos, T. Chan, and R. Molina, "Blind image deconvolution: problem formulation and existing approaches," in Blind image deconvolution: theory and applications, P. Campisi and K. Egiazarian, Eds. Boca Raton: Boca Raton, FL: CRC Press, 2007, ch. 1, pp. 1-41.

[18] W. C. Karl, "Regularization in image restoration and reconstruction," in Handbook of Image and Video Processing, A. C. Bovik, Ed. Orlando, FL, USA: Academic Press, 2000, ch. 3.6, pp. 141-160.

[19] M. A. T. Figueiredo, R. D. Nowak, and S. J. Wright, "Gradient projection for sparse reconstruction: Application to compressed sensing and other inverse problems," IEEE Journal of Selected Topics in Signal Processing, vol. 1, no. 4, pp. 586-597, Dec 2007. 